hydrostatic pressure and so allow growth to continue. It is uncertain how far this applies to extinct forms; many early Palaeozoic cephalopods had thin shells and septa, and the thick shell and septa of Nautilus may be specializations to permit survival at considerable depth, at the price of slower growth.

Ward's book contains a stimulating summary of present knowledge which palaeontologists will find particularly useful. Zoologists who wish to have full information on anatomy and physiology will doubtless turn to Saunders and Landman.

D.T. Donovan is in the Department of Geological Sciences, University College London, Gower Street, London WCIE 6BT, UK.

\section{Reacting to change}

\section{I.W.M Smith}

Molecular Reaction Dynamics and Chemical Reactivity. By Raphael D. Levine and Richard B. Bernstein. Oxford University Press: 1987. Pp.535. Hbk £25, $\$ 39.95$; pbk $£ 15, \$ 18.95$.

Chemical reaction dynamics is concerned with the intricacies of chemical reactions at the molecular level. The field was born in the early 1960s as chemists began to apply molecular-beam techniques and sensitive spectroscopic methods, and as the development of large, high-speed computers enabled them to simulate sufficient numbers of molecular collisions to give their calculations statistical significance.

These studies, and their successors, have revolutionized the way in which chemists visualize chemical changes. We are no longer constrained to think only about how fast chemical species transform at a particular temperature, but we can ask, and often understand, how reactions might be influenced in rate or path by particular forms of energy, how the energy actually released as chemical bonds reform appears in the reaction products, and how the answers to these questions and many others are related to the way in which the forces between the atoms change as they dance around one another during the course of the molecular rearrangement.

Raphael Levine and Richard Bernstein's Molecular Reaction Dynamics, published in 1974 , celebrated the advent of this vigorous area of research and had a richly deserved success. It introduced many students to the field and was a source of guidance and enjoyment to many of its experienced practitioners. Since 1974, however, there have been spectacular advances. Perhaps the most notable of them have resulted from the applications of lasers, especially the tunable dye laser; using techniques such as laser-induced fluorescence and resonance-enhanced multiphoton ionization, it is now possible to prepare and detect species in defined internal states with great sensitivity. The long-heralded marriage of spectroscopic and molecular-beam techniques has not only been consummated but has also become a more equal partnership with the passage of time. There have, too, been parallel advances in beam technology. Developments in the design of nozzle sources have led to beams of greater intensity, defined and definable velocity, and cold internal temperatures, as well as the generation of weakly bound dimers and clusters.

More, surely, is to come, yet the reaction dynamics research community, especially its new recruits, will be grateful to Professors Levine and Bernstein for choosing this moment to revise their earlier text. The style, format and typeface are familiar and give some feeling of déjà vu, but this is misleading. This really is a new book, not just a revision, providing a panoramic and impressively up-to-date view of the subject. It is all done with great enthusiasm and panache, fulfilling the authors' aim of introducing "the language and spirit of the subject".

The organization of the book leads the reader through systems of increasing molecular complexity. After an introductory chapter, explaining what molecular dynamics is about, the concept of molecular collisions and how the dynamics in these collisions reflect the underlying intermolecular forces or potential energy surface are given a central role and are considered in some detail. One disadvantage of this approach is that molecular complexity and conceptual difficulty do not go hand in hand, and it is not until Chapter 4 that the link is established between the microscopic world of molecular collisions and the macroscopic sphere of reaction rates and rate constants. Newcomers to the subject may need some encouragement and guidance to help them connect the material in the early chapters with what is taught in most firstdegree courses.

This is a minor quibble - altogether, the book is an impressive achievement. It brings the reader right to the forefront of the subject, the last chapter bristling with descriptions of new advances in multiphoton effects, molecular clusters, gassurface scattering and stereospecific dynamics. Finally, it is a pleasure to record that this volume has been published at a price that students, and their elders, can afford. It will be widely read.

I.W.M. Smith is a Professor in the Department of Chemistry, University of Birmingham, PO Box 363, Birmingham B15 2TT, UK.

\section{Ups and downs in animal life}

\author{
Tim Clutton-Brock
}

Social Behaviour in Fluctuating Populations. By Andrew Cockburn. Croom Helm: 1988. Pp. 239. £30.

Mammals can be divided into two categories: those that you can see but cannot catch, including most large species; and those that you can catch but cannot see, including most small ones. Because of the problems of visibility, research on the ecology of small mammals has concentrated largely on population demography based on samples of trapped animals. The dramatic population cycles of some species have provided an important stimulus to research in this area, and are at the hub of several controversies, but their causes remain elusive: cycles do not appear to be controlled in any simple fashion by food availability or predation, nor by changes in the frequency of aggressive genotypes in the population. As a result, it is commonly argued that cycles must be driven by behavioural responses to population density, involving reproductive suppression, interference or dispersal. The importance of understanding social organization and reproductive behaviour is consequently widely emphasized.

Cockburn's book provides an excellent review of the behaviour and ecology of cyclical mammals which should be of interest to a wide range of ecologists. Drawing on an extensive knowledge of experimental research on captive rodents, as well as field work on wild populations, he interprets existing knowledge of the life histories, ranging behaviour, mating systems and reproductive physiology of cyclical species within the framework of evolutionary theory. His treatment of the field's controversies is clear and balanced.

Cockburn's principal contribution to arguments over the causes of population cycles is a provocative but well-reasoned rejection of behavioural models. In the final chapter he considers whether a general theory of population cycles is possible, pinning his hopes on more complex models of the interaction between populations and their food supplies which incorporate intra- and inter-seasonal fluctations in food availability. He may be right - but the great diversity of breeding systems and social organization which he documents so clearly carries the obvious implication that demographic processes should be expected to vary widely between species as well.

Tim Clutton-Brock is in the Large Animal Research Group, Department of Zoology, University of Cambridge, 34A Storey's Way, Cambridge CB3ODT, UK. 